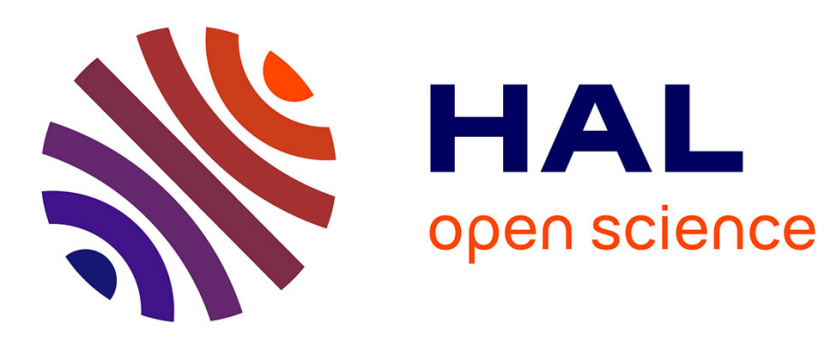

\title{
Interference estimation in an aeronautical ad hoc network
}

\author{
Frédéric Besse, Alain Pirovano, Fabien Garcia, José Radzik
}

\section{To cite this version:}

Frédéric Besse, Alain Pirovano, Fabien Garcia, José Radzik. Interference estimation in an aeronautical ad hoc network. DASC 2011 IEEE/AIAA 30th Digital Avionics Systems Conference, Oct 2011, Seattle, United States. 10.1109/DASC.2011.6095908 . hal-01217569

\section{HAL Id: hal-01217569 \\ https://hal-enac.archives-ouvertes.fr/hal-01217569}

Submitted on 19 Oct 2015

HAL is a multi-disciplinary open access archive for the deposit and dissemination of scientific research documents, whether they are published or not. The documents may come from teaching and research institutions in France or abroad, or from public or private research centers.
L'archive ouverte pluridisciplinaire HAL, est destinée au dépôt et à la diffusion de documents scientifiques de niveau recherche, publiés ou non, émanant des établissements d'enseignement et de recherche français ou étrangers, des laboratoires publics ou privés. 


\title{
INTERFERENCE ESTIMATION IN AN AERONAUTICAL AD HOC NETWORK
}

\author{
Frédéric Besse, Alain Pirovano, Fabien Garcia, ENAC, Toulouse, France \\ José Radzik, ISAE, Toulouse, France
}

\begin{abstract}
Recent research have considered aeronautical ad hoc networks as a possible mean for future aeronautical communications. By introducing interaircraft links, they are supposed to become an alternative to existing solutions based on direct airground or satellite links. In this paper, we propose the use of asynchronous Code Division Multiple Access (CDMA) in aeronautical ad hoc networks. We then present a simulation model developed with OPNET Modeler that estimates the impact of Multiple Access Interference (MAI) on packets delivery. Finally, we give the results of some simulations made with an ATC/AOC traffic model, and with real aircraft positions over the French sky.
\end{abstract}

\section{Introduction}

In the current aeronautical communications context, there is an increasing interest in offering new services aboard civil aircraft. For instance, airlines are looking into new solutions to back up flight data and cockpit voice records during the flight, or to provide more accurate meteorological data to pilots. Being able to offer Internet access to passenger is also a major demand from these airlines companies.

All these services represent new types of airground communications that require a higher capacity than existing systems (such as VDL Mode 2, L-Band satellites for example). In addition to these traditional datalink systems based on a direct airground or satellite link, aeronautical ad hoc networks have recently been proposed as a new solution for future air-ground communications (e.g.: in [1-3]).

The idea of such networks is to introduce wireless links between aircraft. Each aircraft is then seen as a node of the network and is able to act as a router for data sent by other aircraft. Hence, in order to reach a ground station, the data sent by an aircraft may be forwarded several times by other aircraft on its path. An aeronautical ad hoc network is a selfconfiguring, self-healing network and is based on a light ground infrastructure. Figure 1 shows the topology of such a network. It can be seen that, even if some aircraft are outside the coverage area of the ground stations, they are nevertheless able to communicate with them via other aircraft.

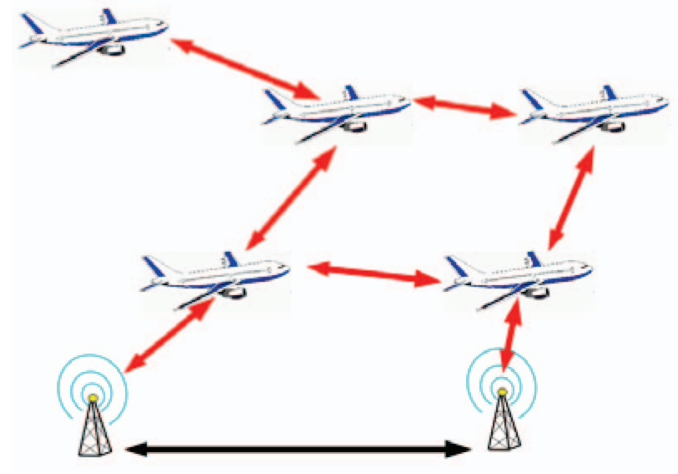

Figure 1. Topology of an Aeronautical Ad Hoc Network

\section{Related Work}

Aeronautical ad hoc networks have been previously studied in some projects, for example in the ATENAA project [4] or in the NewSky project [5]. In this latter, the feasibility of an aeronautical ad hoc network over the North Atlantic corridor has been demonstrated in [6]. It is based on a list of flight plans and trajectories which are interpolated between departure and arrival airports with great circle arcs. But in this study, the link model is not really addressed. A simplified communication link model is used. They consider that if the distance between two aircraft is smaller than a given transmission range, a connection is available and no error due to the channel can occur.

Obviously the routing protocol is very important in ad hoc networks, especially in aeronautical ad hoc networks where we may have a very dynamic topology because of the high speed of aircraft. In [1], [2], a routing protocol is proposed for aeronautical ad hoc networks. It takes into account the relative 
aircraft velocity to create stable clusters. The main goal of this approach is to maximize links duration.

In the ATENAA project, another routing protocol named ARPAM (Ad hoc Routing Protocol for Aeronautical Mobile Ad Hoc Networks) and based on AODV has been proposed in [7]. In this study, they use Ka-Band directional antennas for the links between aircraft. As the issue of pointing and tracking an inflight aircraft represents an expensive and complex approach, we have decided to use only omnidirectional antennas on-board the aircraft.

In [8], we demonstrate the feasibility of an aeronautical ad hoc network over the French sky. This study is based on actual aircraft positions data provided by DSNA (the French provider in charge of air navigation services). We present a simulation tool developed to assess the performance of an aeronautical ad hoc network in term of connectivity and offered capacity. For example, we show the influence of the communication range on the connectivity.

In [9], we estimate the available throughput per aircraft and we propose the use of an aeronautical ad hoc network as an access channel to reach a ground station from the aircraft. The ground to aircraft link is based on a geostationary satellite. The available throughput per aircraft estimation is based on a link budget of the connection between aircraft. We consider a free space propagation model and we establish the relationship between the connection length and the available throughput. Then we assume that a routing protocol provides to each aircraft the shortest path to the closest ground station. All aircraft are supposed to send data constantly. The available throughput on each link is then fairly divided among all aircraft using this link.

\section{Network Model}

In this paper, we propose a new simulation model for CDMA aeronautical ad hoc network. It has been developed with OPNET Modeler [10], which is a discrete event simulator for all kind of networks.

The aim of the model is to simulate aeronautical ad hoc network in a realistic way. In our model, we can choose the aircraft number, and also the ground station number. We take into account actual aircraft positions in the French sky.
Moreover, we can define the traffic generated by each aircraft. We can study the performances of the aeronautical ad hoc network with different traffic profiles, meaning different applications. We are also able to try several routing algorithms, with different communication ranges, different ground station positions...

Figure 2 shows a screenshot of our model in OPNET Modeler.

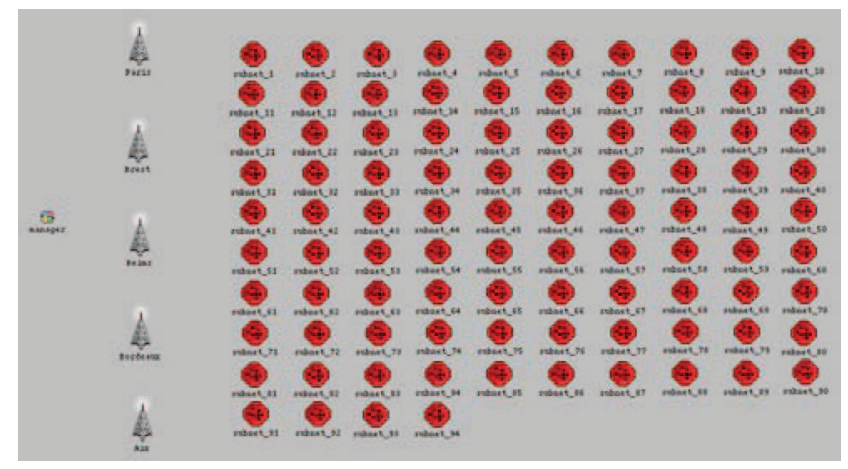

Figure 2. Our OPNET Simulation Model

The model is composed of three types of nodes. We have aircraft, ground stations and a manager.

The manager is used to update aircraft positions from an input data file. It also computes global information for the routing protocol, such as the Dijkstra algorithm. It avoids the computation of these information by all aircraft. Thus it saves simulation time. Finally, the manager collects all the simulations global statistics.

Then, the two other types of nodes are aircraft themselves and ground stations. They are quite different because for now, we consider that all aircraft only send data to ground stations. Then, we only study the access link from the aircraft to the ground. The return channel from the ground the aircraft is supposed to be a satellite or another ad hoc network with a different carrier frequency. We only choose the return channel delay in our simulation parameters.

The base station node is then simpler than the aircraft one because it can only receive data, whereas aircraft can of course send data, but also receive data from other aircraft. Figures 3 and 4 show respectively the architecture of aircraft and base station node models. 


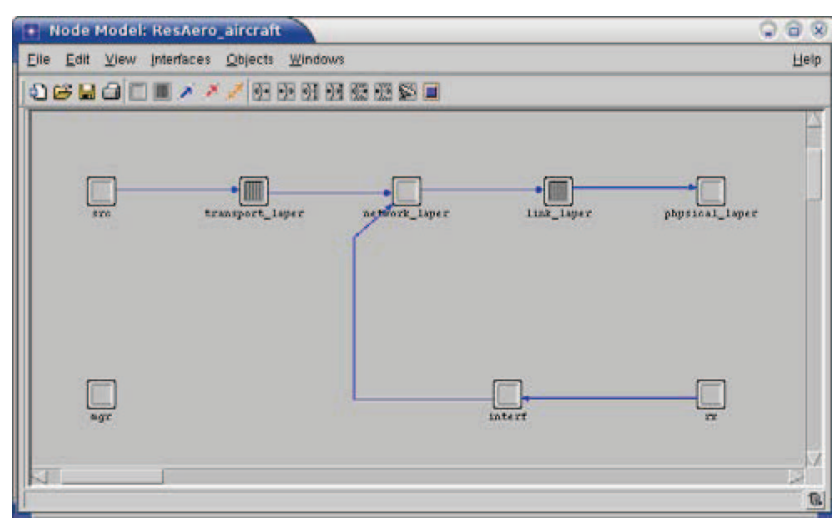

Figure 3. Aircraft Node Model Architecture

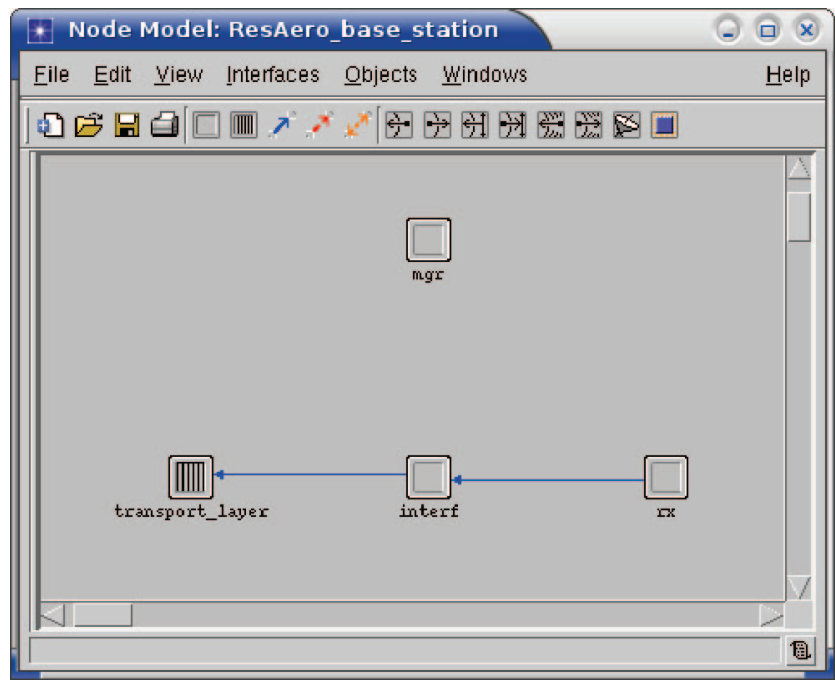

Figure 4. Base Station Node model Architecture

We will now detail the different node models layers.

\section{Physical and Data Link Layers}

In an aeronautical ad hoc network, we have a wireless channel for the communications between aircraft, and between aircraft and ground stations. Thus, we have to share this channel among all users, meaning all aircraft in the sky.

Several multiple access methods exist. First, we have Time Division Multiple Access (TDMA), which has been proposed for aeronautical ad hoc network in [11]. Then, there are Frequency Division Multiple Access (FDMA) and Code Division Multiple Access (CDMA). But we think that TDMA and FDMA will be quite inefficient at high load because of the high aircraft number. That's why we decided to focus on CDMA.
CDMA is widely used in the third generation of cellular networks. In such networks, it has been shown in [12] that it provides up to six times the capacity of TDMA and FDMA-based solutions. That is also why we decide to use CDMA.

The feasibility of fully asynchronous CDMA wireless ad hoc networks has been investigated in [13]. They have a Matlab-based simulation model to study multi-users interferences. They study the influence of several parameters, including node number, distance between nodes, chip length, signal propagation, connection per node number. But they simulate up to 200 nodes, which is not enough for our study as we have about 600 aircraft flying in the French sky during the day. Moreover, they use a network model with static connections, which is unrealistic in an aeronautical ad hoc network, considering the high speed of aircraft.

On the other hand, IEEE 802.11 is widely used in wireless communication. It has become the de facto standard for medium access control in wireless local area networks (WLAN). That's probably why many studies on ad hoc networks are based on IEEE 802.11, especially for wireless sensor networks.

But the transmission range of IEEE 802.11 is typically of some meters or some tens of meters. In aeronautical ad hoc networks, we have shown in [8] that a transmission range of about $100 \mathrm{~km}$ is required to ensure a high percentage of connected aircraft. That's why IEEE 802.11 can not be used as it is in aeronautical ad hoc networks.

Moreover, the channel access method implemented in IEEE 802.11 is CSMA/CA. In this method, a carrier sensing scheme is used. It means that a user who wants to transmit data has first to listen to the channel to determine whether it is busy or not. If the channel is sensed as free, the user is allowed to transmit data; otherwise it has to defer its transmission. As a consequence, CSMA/CA supports only one transmission at a time within the transmission range.

But as we have a high transmission range and a high density of aircraft over the French sky, in the USA or in the North Atlantic Corridor, we always have many aircraft within the transmission range. It means that with CSMA/CA, the overall available throughput will be low because when an aircraft will 
send data, the channel will be busy for many aircraft nearby.

CDMA increases the network capacity because it allows multiple successful transmissions at the same time. Indeed, even if there are collisions, all collided packets can be successively received by the intended receiver. But this can only be done within the limits of Multiple Access Interferences (MAI), which are the major factor limiting the performance of CDMA systems.

We will now rapidly describe the spread spectrum principle used in CDMA, and then we will describe multiple access interferences.

\section{Spread Spectrum Principle}

CDMA employs spread spectrum technology. In this paper, we have chosen direct sequence spread spectrum.

We assume that each aircraft has a unique pseudo-random spreading code $c(t)$ of bandwidth $f_{c}=1 / T_{c}$ bits $/ \mathrm{s}$. This spreading code is a binary sequence that statistically appears as a random sequence, but that can be exactly reproduced by the receiver, according to mathematical rules.

When an aircraft want to send a data digital signal $d(t)$ of bandwidth $f_{d}=1 / T_{d}$ bits/s, it spreads this signal with the spreading code $c(t)$. Then, it sends the spread signal $s(t)=c(t) \cdot d(t)$ to the intended receiver.

$N=T_{d} / T_{c} \gg 1$ is the processing gain. As a consequence, the spread spectrum signal $s(t)$ occupies a much greater bandwidth than the original data signal $d(t)$. The data signal energy is also spread on all the available bandwidth, meaning that the signal is transmitted at a very low power level. Thus it has a very low probability of detection and interception, and is very resistant to interference and jamming. That's why spread spectrum was firstly developed for military applications.

At the receiver, by multiplying the received signal by the same spreading code $c(t)$ exactly reproduced locally, we can despread the received signal and recover the original data signal $d(t)$.

Figure 5 shows the principle of direct sequence spread spectrum.

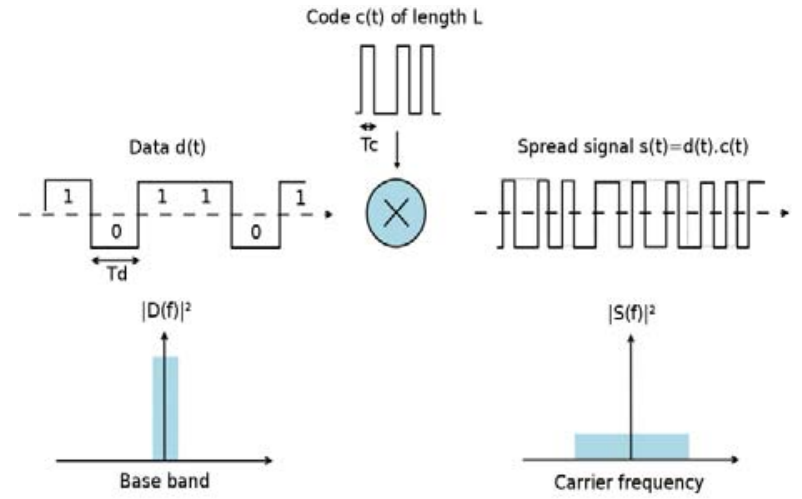

Figure 5. Direct Sequence Spread Spectrum Principle

\section{Multiple Access Interference}

CDMA allows the use of several independently coded signals in the same channel. But to obtain perfectly orthogonal signals, a distinct code for each signal is needed, and above all a perfect synchronization between the transmitter and the receiver [14].

This point is particularly important. That's why CDMA based networks were firstly developed with base stations with dedicated pilot channel to facilitate synchronization. But in ad hoc networks, there isn't any infrastructure to ensure synchronization. Thus, we assume a fully asynchronous CDMA aeronautical ad hoc network.

Unfortunately, there is no orthogonal codes family for asynchronous CDMA. The spreading codes are statistically uncorrelated but multiple simultaneous users result in Multiple Access Interferences (MAI). Each connection between two aircraft causes interferences to all other simultaneous connections in the system. Links quality and packets reception can then be affected.

For now, in existing studies on aeronautical ad hoc networks, it is considered that as soon as two aircraft are closer than a given distance, typically the optical range, a connection between them can be established. The link quality is then given by the length of the connection. Indeed, the longest the connection is, the lowest is the received power. The number of simultaneous transmissions is not taken into account.

In our model, multiple access interferences are estimated by aircraft and ground stations for each 
received packet to determine whether it is correctly received or not. Figure 6 shows the architecture of the Multiple Access Interference Estimation process.

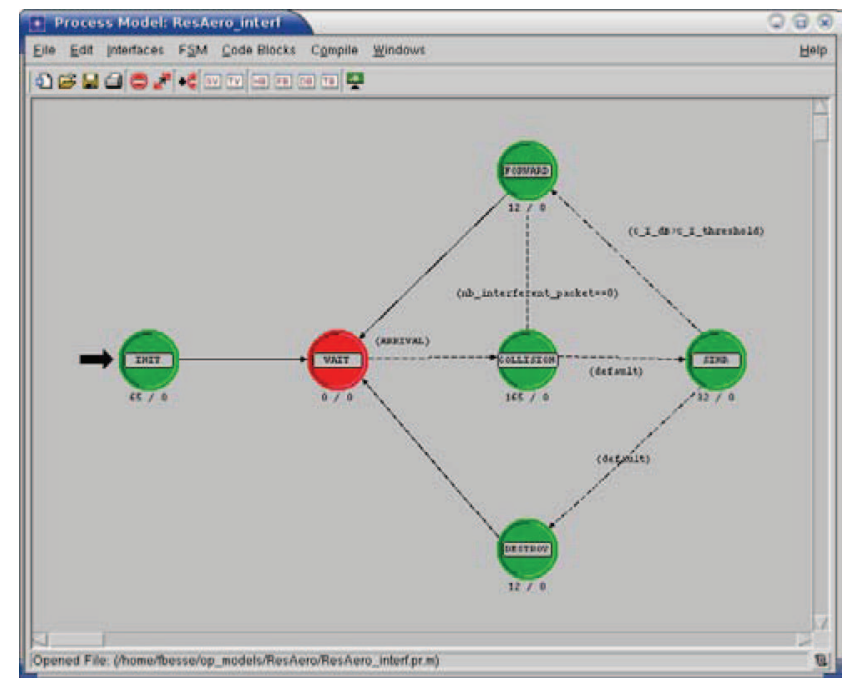

Figure 6. Multiple Access Interference Estimation Process

First of all, we determine if the received packet has been collided, considering all pending packets that have been sent before. If no collision occurs, the received packet is transmitted to upper layers. Otherwise, we obtain a list of $n$ interfering packets. We compute the signal to noise and interference ratio (SINR) of the received packet [15]:

$$
\operatorname{SINR}_{i j}=\frac{P_{i} \cdot L_{i j}}{\frac{1}{N} \sum_{k=1}^{n} P_{k} \cdot L_{k j}+\sigma^{2}}
$$

where $P_{i}$ is the transmission power of node $i, L_{i j}$ the channel attenuation from node $i$ to node $j$, the received power at node $j$ is then $P_{i} . L_{i j}, n$ is the number of interfering packets, $N$ is the processing gain, $\sigma^{2}$ is the thermal noise.

$L_{i j}$ is computed with the respective positions of node $i$ and node $j$, assuming a free space propagation model. This is justified because we consider aircraft to aircraft communications in direct line of sight and atmosphere attenuation is neglected. As omnidirectional antennas are used, we have:

$$
L_{i j}=\left(\frac{\lambda}{4 \pi D_{i j}}\right)^{2}
$$

where $\lambda$ is the wavelength and $D_{i j}$ the distance between the transmitter and the receiver. The system frequency is set to $2 \mathrm{GHz}$.

Finally, the received packet SINR is compared to a threshold, which is a parameter of our simulation. It can be computed according to the system requirements in terms of bit error rate. If the SINR is higher than the threshold, the packet is considered as correctly received and transmitted to higher layers. Otherwise, the received packet is destroyed.

\section{Code Distribution}

Another issue has to be addressed by the physical and data link layers in CDMA aeronautical ad hoc networks. Indeed, each aircraft is supposed to be identified by a unique pseudo random code. Two main issues come up there. First of all, many codes are required. In a typical day of June 2007, more than 9000 different aircraft have flown over the French sky during a whole day. Even if they are not flying at the same time, there are always more than 400 aircraft in the sky between 6 a.m. and 8 p.m. Figure 7 shows the number of inflight aircraft over France on June, $22^{\text {th }} 2007$.

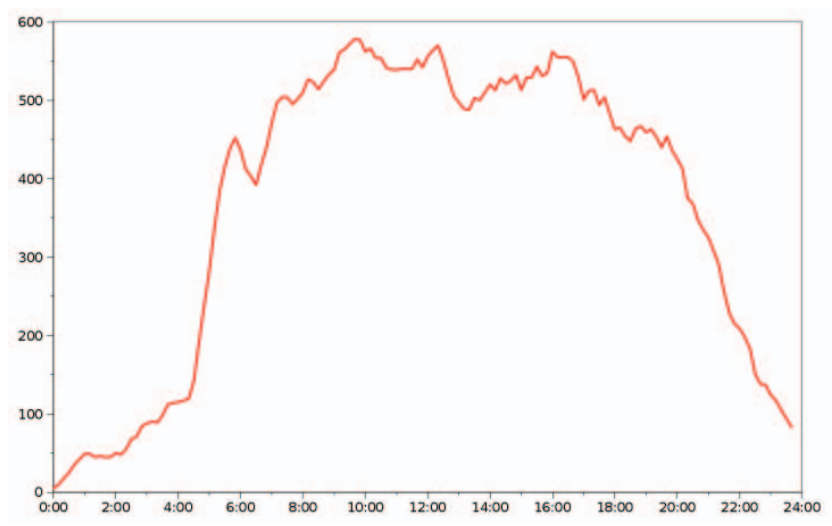

Figure 7. Number of Inflight Aircraft

In asynchronous CDMA, a well-known family of codes is Gold codes. They are suitable for asynchronous CDMA because they have a trivalent cross correlation function, which makes it easy to determine the maximum of the correlation between the received signal and the code generated locally. Gold codes are used for example in the Global Positioning System (GPS). The code length L has to be chosen considering the available bandwidth and the required data throughput. The available number 
of Gold Code of length $\mathrm{L}$ is then $\mathrm{L}+2$. If the number of inflight aircraft is greater than $\mathrm{L}+2$, it is impossible to provide a unique code to each aircraft.

That's why we may need a code allocation protocol to dynamically assign code to aircraft. In the literature, some code allocation protocols have been proposed, for example in [16]. Most of them assign codes to aircraft on demand, while ensuring that neighbor aircraft have different codes.

Now, we can assume that each aircraft has a pseudo random code, even if it is not unique in the network. Here comes up the second issue. Indeed, as we have seen in spread spectrum, the receiver has to reproduce locally the spreading code used by the transmitter. It means that the intended receiver needs to know the transmitter code. Before establishing a communication between two aircraft, we need a handshake between them to exchange the spreading code. They can just exchange a code ID because we can assume that aircraft are not memory limited, and that each aircraft has access to the spreading codes list. Therefore they don't need to exchange the whole code, but just an ID, which is supposed to be smaller.

To exchange this control information, we assume that we have a common code for all aircraft. This common code will also be used for the signalization and for the routing. For this common channel, a slow rate will be used in order to be less subject to interference and to have a higher communication range. If the common channel suffers from too many multi-users interferences, we can also use a dedicated frequency channel, as suggested in [17].

The last thing is to agree on the code used for the communication. Basically, this choice can be receiver-based or transmitter-based. We can use the transmitter's code or the receiver's one. By using the receiver's code, aircraft will always receive data on their own code. The receiver will be simple but primary collisions can happen when two aircraft want to send data to the same one. Indeed, they will use the same code. That's why it's better to use the transmitter's code. Indeed, collisions of packets with the same spreading code are impossible. Moreover, it becomes easy for an aircraft to broadcast data. The receiver becomes a bit more complex because it may have to listen to several different spreading codes at the same time. But it is not an issue for an aircraft.

\section{Network Layer}

For the routing algorithm, we considered for now that each aircraft is connected to the closest ground station with the shortest path, given by the Dijkstra algorithm. The maximum distance to establish a connection between two aircraft is a simulation parameter.

We consider a graph, whose nodes are aircraft and ground stations, and whose edges are the available connections between them. We assume that a connection is always available between all pairs of ground stations. The weight of each edge in the graph is its length. Then, the Dijkstra algorithm is used to find in this graph the shortest path from each aircraft to a ground station. It gives for each connected aircraft a path to the closest ground station.

In our model, to avoid the computation of the Dijkstra algorithm by each aircraft, it is only done by the manager node at each aircraft position update. Then, all aircraft are updated with their new next hop aircraft along the path to the closest ground station. Each aircraft has to send its traffic to this next hop aircraft.

\section{Transport Layer}

At the transport layer, a retransmission system is implemented in our model. Both an acknowledgment mechanism and a retransmission timer are used.

Acknowledgments (ACK) are only send by ground stations as we only consider for now communications between aircraft and ground stations. When a ground station receives a packet, it sends an acknowledgment to the packet source with the packet sequence number. As we don't study yet the return channel, this ACK is directly sent to the source with a given delay, which is a parameter of our simulations. The ACK packet doesn't go through the ad hoc network. As a consequence, we can't have loss of ACK packets.

The retransmission timer is set to the average of the round trip time (RTT) multiplied by two. The maximum retransmissions number of each packet is a simulation parameter. After the maximum number of attempts to retransmit a packet, and if no acknowledgment is received, the packet is definitively destroyed and considered as lost. 
In our system, the reliability is only ensured at the transport layer, meaning between the source and the ground station. We have chosen not to implement reliability at the data link layer, meaning for each aircraft to aircraft connection, because we think that the overhead generated by all needed acknowledgments will degrade too much the overall available throughput.

\section{Traffic per Node}

The traffic per node is a simulation parameter. We can choose for example the distributions of packets size and inter arrival time. We can choose different laws (uniform, Poisson...). The results of another OPNET traffic model can also be directly injected.

\section{Simulations}

\section{Hypothesis}

\section{Aircraft Positions}

We use the same aircraft position data that we have used in [8]. They have been provided by DSNA, the French provider in charge of air navigation services. We have several days of traffic with the actual aircraft positions each fifteen seconds over the French sky. For these simulations, we choose June, $22^{\text {th }} 2006$.

\section{Ground Stations}

In this paper, we have two scenarios for ground stations. First, we assume that there is only one ground station in the French sky. This station is located in Paris. Then, we have a second scenario with five ground stations.

In a previous study we have made in the French Sky, we have shown that the location of ground stations hasn't a great impact on the network performance. It is probably due to the aircraft density in the French sky, which is quite homogeneous. Finally, we decide to use the five French "en route" air traffic control centers as ground stations.

\section{Aircraft and Ground Stations Ranges}

In previous studies on aeronautical ad hoc networks, the aircraft range is often set to maximum available range, which is the optical range. It is computed for two aircraft flying at an average cruise altitude of about $10 \mathrm{~km}$ (FL300), which gives about
$680 \mathrm{~km}$. This leads to a very good connectivity, meaning a high percentage of connected aircraft.

On the other hand, the longest the connections between aircraft are, the lowest is the available throughput. In [9], we show that a transmission range between 100 and $200 \mathrm{~km}$ was a good tradeoff between connectivity and performance. In our simulations, we have a first scenario with a $100 \mathrm{~km}$ range, and a second one with $200 \mathrm{~km}$. This allows us to see the influence of the communication range on the system performances.

\section{Traffic Model}

In this paper, we simulate Air Traffic Control (ATC) and Airlines Operational Communications (AOC). According to a study [18] made by Eurocontrol and the University of Salzburg on VDL 2 , we have chosen a packet size uniformly distributed between 32 and 265 bytes, and an inter arrival time distribution following an exponential law, with an average of $38.46 \mathrm{~s}$, meaning 1.56 messages per minute and per aircraft on average for both ATC and AOC traffic.

\section{Retransmissions Number}

We have two scenarios. In the first one, we assume no retransmission at all. It allows us to see the actual network performance without any retransmission mechanism. In the second time, the retransmission mechanism is activated. The maximum retransmissions number of each packet is set to 16 .

\section{Performance Metrics}

For ad hoc networks optimization, performance metrics are a key issue. For example, many studies in the literature are focused on energy consumption minimization, which is a performance metric for ad hoc network. Indeed, first applications of ad hoc networks were designed for small electronic devices, for example with Bluetooth, or for sensors networks. These devices are by nature energy-limited. But in our case, we assume that aircraft are not energylimited. Thus, energy consumption is not the main of our interest.

For us, it is for example more relevant to optimize the overall available throughput, especially if we want to support heavy traffic load in the network, such as the one generated by providing an Internet access to aircraft passengers. 
In this paper, we focus on the number and the percentage of packets lost because of multiple access interferences. We also study packets delays and hop counts.

\section{Influence of the Processing Gain}

In this part, simulations are performed with one base station located in Paris, without the retransmission mechanism and with a $100 \mathrm{~km}$ aircraft range.

As in all CDMA-based system, the processing gain choice is a major issue in our aeronautical ad hoc network. As a remember, the processing gain can be computed as the ratio between the code and the data rates. The code rate is limited by the available bandwidth. In all our simulations, we always consider the same spreading code rate of $20 \mathrm{Mcps}$.

Then, we can choose the processing gain by choosing the data rate, meaning the capacity of links between aircraft. But this choice is not very simple. If a high data rate is used, the requested time to send and receive data is short and we are less subject to collisions. But the processing gain is low. The system is less resistant to interferences. On the other hand, if a low data rate is chosen, the processing gain is high, meaning that we will overcome more easily from collided packets. But as it takes more time to send and receive packets, there are more collisions. Moreover, in case of heavy traffic, the system suffers from congestion more rapidly.

A tradeoff has to be made for the processing gain. In fact, this tradeoff is highly related with the traffic load. We made some simulations with our traffic model with a data rate varying from $100 \mathrm{kbps}$ to $2 \mathrm{Mbps}$.

Figure 8 shows the percentage of packets lost because of interferences. We can see that the optimum spreading factor is obtained for a data rate of about $500 \mathrm{kbps}$, where we have about $0.41 \%$ of packets loss.

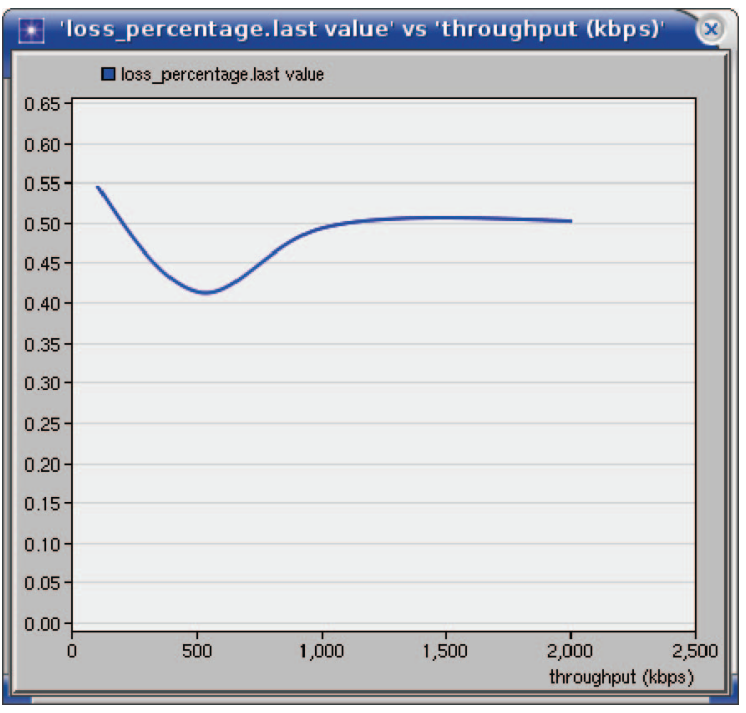

Figure 8. Influence of the Spreading Gain on the Packets Loss Percentage

In fact, the signal to noise and interference ratio of all received packets is expected to be higher if the data rate is low because the processing gain increases. But when we look at the number of collisions and the number of interfering nodes within each collision in figures 9 and 10 , we can see that the collisions number is inversely proportional with the data rate. Indeed, the collision probability is proportional to packet reception duration. For the same reason, the number of interfering packets in each collision also increases when the data rate decreases.

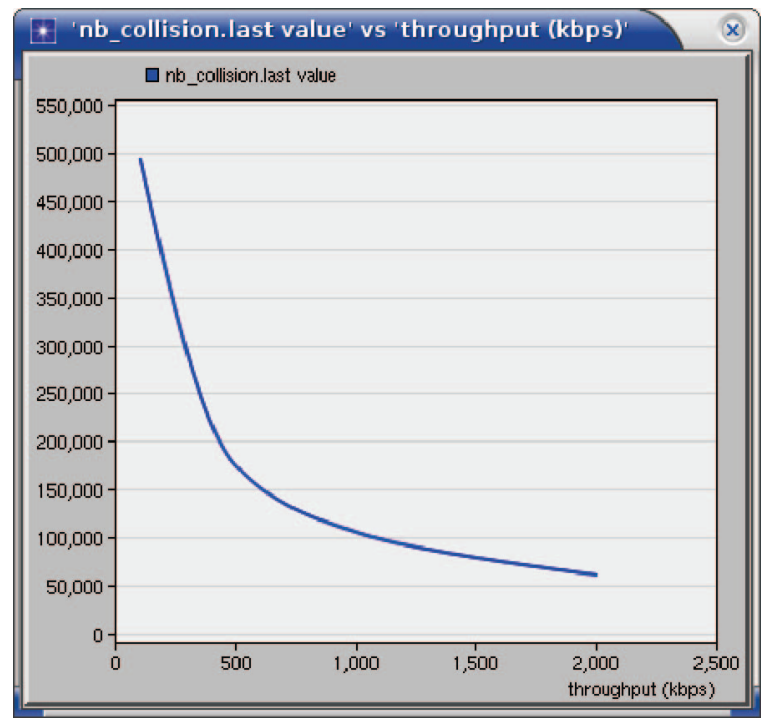

Figure 9. Influence of the Spreading Gain on the Collisions Number 


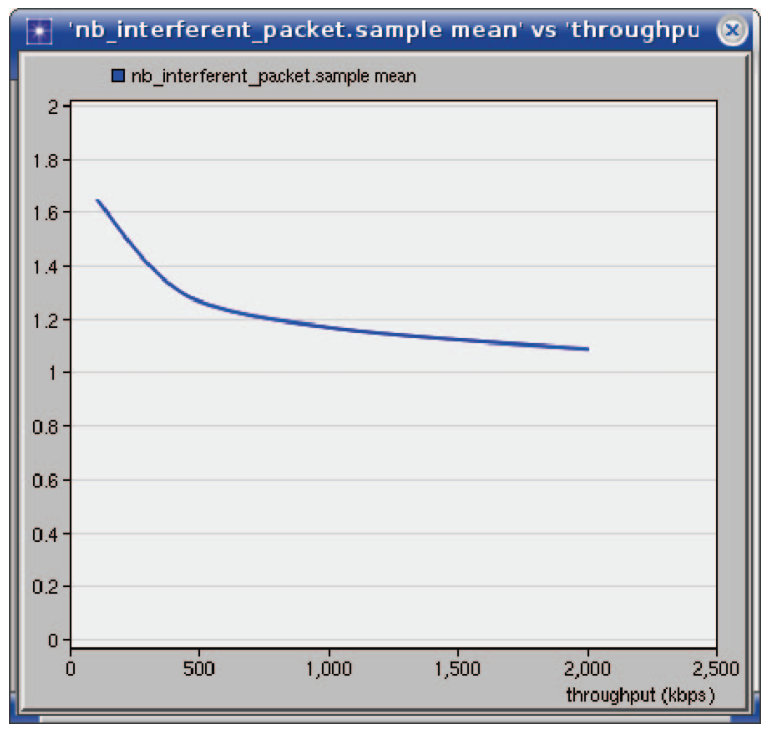

Figure 10. Influence of the Spreading Gain on the Number of Interfering Packets in each Collision

\section{Influence of the Communication Range}

In this part, we want to optimize the average packets delay. One solution is to increase the communication range so that we have longer hops, and then less hops. Simulations show that for a 200 $\mathrm{km}$ transmission range, we obtain an average of 3 hops for a packet to reach the ground station, compared with 5.5 hops with a $100 \mathrm{~km}$ range.

Figure 11 shows the communication range influence on packets delay.

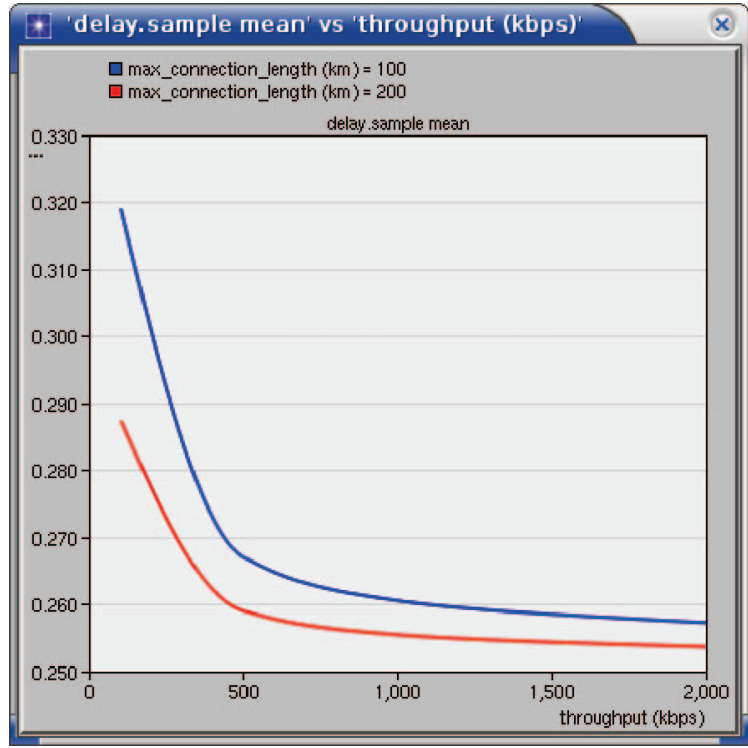

Figure 11. Influence of the Communication Range on Packets Delay
The y graph scale starts at $250 \mathrm{~ms}$, which is the chosen return channel delay. It represents a geostationary satellite. We can see that received packets delays decrease when the communication range increases. We can also see that the average delay severely increase when the data rate decreases in both cases. Indeed, when the data rate decreases, it takes more time to send a packet and a little congestion appears.

Moreover, by increasing the communication range, the ground station has to handle with a higher connections number. When the communication range is increased from 100 to $200 \mathrm{~km}$, the number of aircraft directly connected to the ground station increases from 52 to 117 aircraft on average during the day. The number of packet destroyed by the ground station because of multiple access interferences increases by more than $100 \%$. The lost packets percentage increases from $0.51 \%$ to $0.68 \%$, considering a data rate of $500 \mathrm{kbps}$.

That's why we keep a $100 \mathrm{~km}$ communication range for the following simulations.

\section{Influence of the Ground Stations Number}

Another way to reduce packets delay is to add ground stations. But we have to keep in mind that aeronautical hoc networks must have a little ground stations number, in order to be competitive with cellular solutions. We make simulations with 5 ground stations, compared to the original scenario with only one. Table 1 shows the results about average hop count, packets delay and loss percentage of these two scenarios, considering a data rate of 500 kbps.

Table 1. Influence of the Ground Stations Number

\begin{tabular}{|l|l|l|}
\hline Parameters & One station & Five stations \\
\hline $\begin{array}{l}\text { Average hop } \\
\text { count }\end{array}$ & 5.5 & 2.8 \\
\hline $\begin{array}{l}\text { Average packet } \\
\text { delay (ms) }\end{array}$ & 267 & 259 \\
\hline $\begin{array}{l}\text { Loss } \\
\text { percentage (\%) }\end{array}$ & 0.41 & 0.07 \\
\hline
\end{tabular}

With five stations, the average packets delay in the aeronautical ad hoc network decreases from 17 to $9 \mathrm{~ms}$, as we have a fixed $250 \mathrm{~ms}$ return channel delay. The loss percentage is also decreased because the load is shared among the five ground stations. 
Finally, we obtain a delivery ratio of $99.93 \%$, which is very good, knowing that there is no retransmission mechanism. As soon as a packet is destroyed by an intermediate aircraft, it is definitively lost.

\section{Influence of the Retransmission Mechanism}

In this part, we activate the retransmission mechanism. The maximum retransmissions number of a packet is set to 16 .

Figure 12 shows that very good results are obtained. Considering only one ground station, we have no packets loss with data rates from $100 \mathrm{kbps}$ to 2 Mbps.

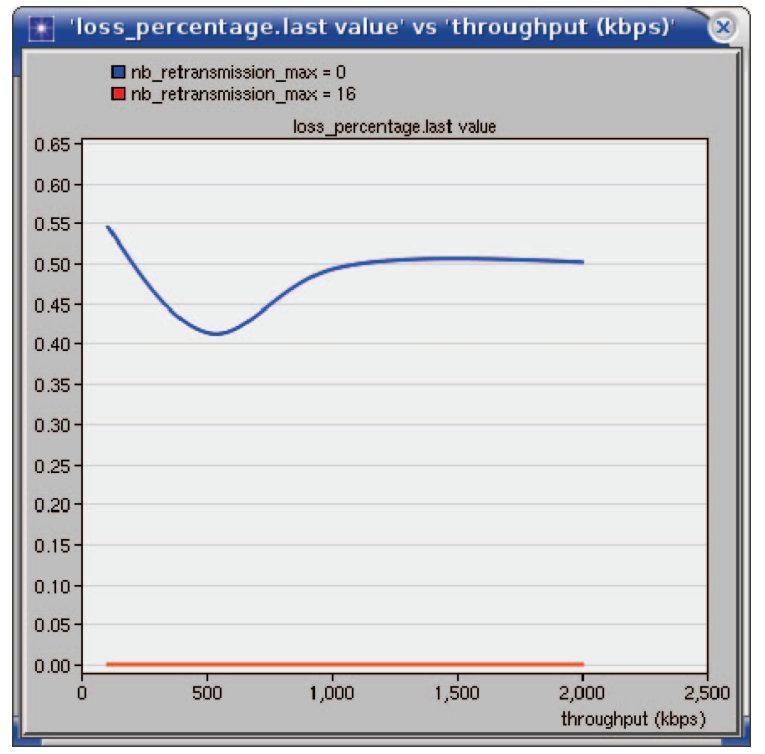

Figure 12. Influence of the Retransmission Mechanism on the Packet Loss Percentage

In fact, if we look closer to the simulation results, we can see that only three retransmissions are required.

\section{Conclusion and Future Works}

In this paper, we have shown that fully asynchronous CDMA can be used in aeronautical ad hoc networks. Simulations made with real aircraft positions over the French sky show a 100\% packet delivery ratio can be reached with only one ground station and a simple retransmission mechanism on such a network. Moreover, we have shown that with only five ground stations, we obtained a $9 \mathrm{~ms}$ average packets delay for the link between aircraft and ground stations.
These results are very encouraging, though for future works we will have to study performances of our proposed network at higher traffic load. We also plan to optimize the routing algorithm and to study its impact on the performances of the network.

\section{References}

[1] E. Sakhaee, A. Jamalipour, and N. Kato. Aeronautical Ad Hoc Networks. In Wireless Communications and Networking Conference (WCNC), 2006. IEEE, volume 1, pages 246 -251, 362006.

[2] E. Sakhaee and A. Jamalipour. the Global inFlight Internet. Selected Areas in Communications, IEEE Journal on, 24(9):1748 -1757, sept. 2006.

[3] K. Karras, T. Kyritsis, D. Yannis, and G. Bogdos. Applicability of Mobile Ad Hoc Networks in an Aeronautical Environment. International Conference RoEduNet,2007.

[4] Amirfeiz, M., "ATENAA Project : Advanced Technologies for Networking in Aeronautical Applications," 2006.

[5] Schnell, M. and Scalise, S., "Newsky - A Concept for Networking the Sky for Civil Aeronautical Communications," 25 ${ }^{\text {th }}$ Digital Avionics Systems Conference (DASC), 2006 IEEE/AIAA, 15-19 2006, pp. $1-6$.

[6] Medina, D., Hoffmann, F., Ayaz, S., and Rokitansky, C.-H., "Feasibility of an Aeronautical Mobile Ad Hoc Network Over the North Atlantic Corridor," Sensor, Mesh and Ad Hoc Communications and Networks, 2008. SECON '08. 5th Annual IEEE Communications Society Conference on, 16-20 2008, pp. 109 -116.

[7] Michael Iordanakis, Dimitrio Yannis, Kimon Karras, Gergios Bogdos, Georgios Dilintas, Massimiliano Amirfeiz, Giorgio Colangelo, Stefano Baiotti, "Ad Hoc Routing Protocol for Aeronautical Mobile Ad Hoc Networks"

[8] F.Besse, A.Pirovano, F.Garcia, J.Radzik, "Aeronautical Ad Hoc Networks : a new Datalink for ATM", 9th Innovative Research Workshop \& Exhibition, SESAR WPE, 2010

[9] F.Besse, A.Pirovano, F.Garcia, J.Radzik, "Wireless Ad Hoc Networks Access For Aeronautical Communications", 28th AIAA 
International Communications Satellite Systems Conference (ICSSC), 2010, Anaheim, CA

[10] Opnet Website. http://www.opnet.com

[11] Felix Hoffmann, Daniel Medina, Adam Wolisz, "Optimization of Routing and Gateway Allocation in Aeronautical Ad Hoc Networks Using Genetic Algorithms", 2011

[12] K.S. Gilhousen, I.M. Jacobs, R. Padovani, A.J. Viterbi, L.A. Weaver, C.E. Wheatley III, "On the Capacity of a Cellular CDMA System", IEEE Transactions on Vehicular Technology, 1991.

[13] Bernd Thallner, Günther Gridling, "Simulation Of a Wireless CDMA Ad Hoc Network"

[14] J. G. Proakis. Digital Communications. McGraw-Hill, Inc., 2001.

[15] Aylin Yener, Shalinee Kishore, "Distributed Power Control and Routing For Clustered CDMA Wireless Ad Hoc Networks", 2004

[16] Saurabh Srivastava, Sachee Tripathi, Dheeraj Sanghi, Ajit K. Chaturvedi, "A Code Allocation
Protocol for Maximizing Throughput in CDMA based Ad Hoc Networks", 2003

[17] Alaa Muqattash and Marwan Krunz, "CDMABased MAC Protocol for Wireless Ad Hoc Networks", MobiHoc 2003

[18] Eurocontrol \& University of Salzburg, "Advanced VDL 2 Capacity Analysis through Simulations", 2003

\section{Acknowledgements}

Financial support of this research by ENAC and THALES AV. is gratefully acknowledged.

\section{Email Addresses}

For further information, authors can be contacted at frederic.besse@enac.fr, alain.pirovano@enac.fr, fabien.garcia@enac.fr and jose.radzik@isae.fr.

30th Digital Avionics Systems Conference

October 16-20, 2011 